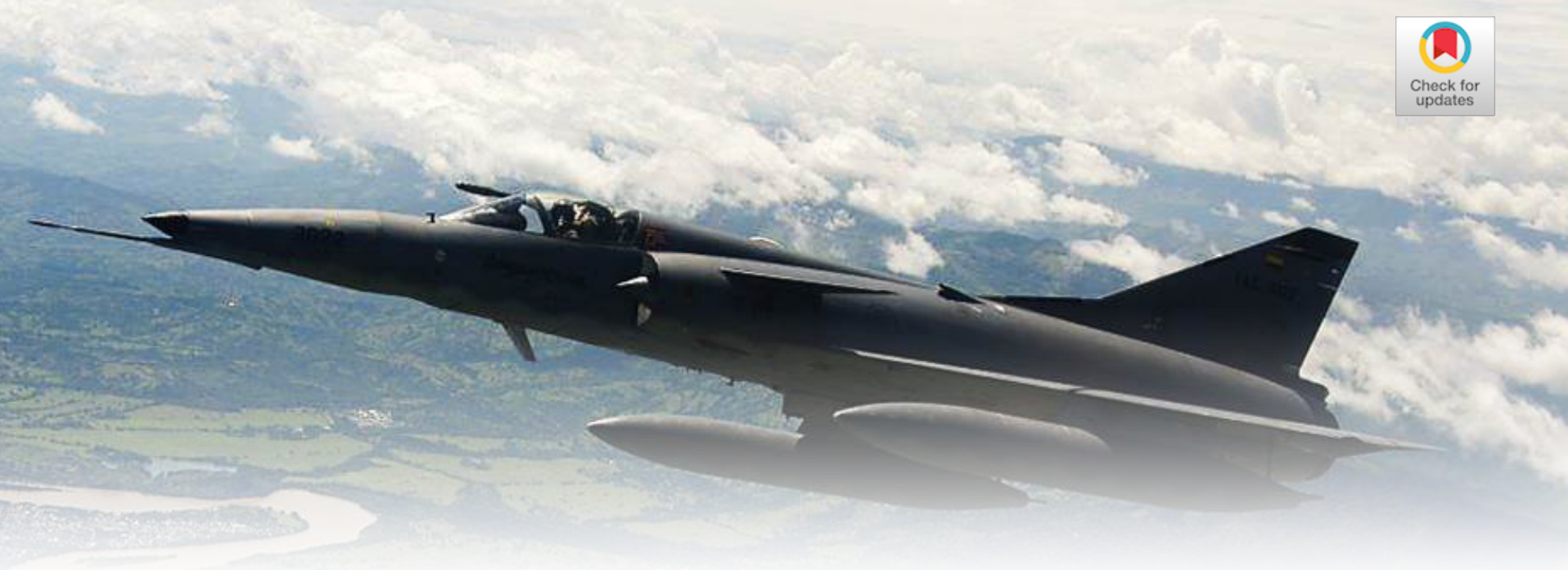

\title{
Los intereses estratégicos de Argentina en el marco institucional de Mercosur
}

CIENCIA Y PODER AÉREO

ISSN 1909-7050 | E-ISSN 2389-2468 |Volumen 9 | Enero-Diciembre de 2014 | Colombia | Pp. 67-74

Recibido: 13/08/2014

Aprobado evaluador interno: 15/09/2014

Aprobado evaluador externo: 09/11/2014

'Artículo de reflexión asociado al proyecto de investigación Prospectivas en Seguridad y Defensa para Colombia: Análisis del entorno América Latina 2001-2013, vinculado al grupo de investigación Centro de Gravedad del Centro de Estudios Estratégicos en Seguridad y Defensa -CEESEDEN- de la Escuela Superior de Guerra. Investigador principal: Pedro Pablo Moreno Jiménez. co-investigadores: Ricardo Esquivel Triana, Juan Pablo Gómez Azuero, Humberto Alarcón Ortiz, Andrés Mateus Rojas. El autor agradece a la institución el apoyo brindado.

${ }^{2}$ Reflection article associated with the research Project: Prospective in Security and Defense for Colombia: Analysis of Latin America context 2001-2013, linked to the Center of Gravity of the Center of Strategic Studies in Security and Defense-CEESEDEN research group - of the War College. Principal Researcher: Pedro Pablo Moreno Jimenez. Co-researchers: Ricardo Esquivel Triana, Juan Pablo Gómez Azuero, Humberto Alarcón Ortiz, Andrés Mateus Rojas. The author acknowledges the institution for the support provided.

${ }^{3}$ Magíster en Relaciones y Negocios Internacionales de la Universidad Militar Nueva Granada, Especialista en Estudios Políticos de la Universidad EAFIT, Especialista en Seguridad y Defensa de la Escuela Superior de Guerra y Profesional en Gerencia de la Seguridad y Análisis Sociopolítico de la Escuela de Inteligencia del Ejército. Docente de la Maestría en Seguridad y Defensa Nacionales de la Escuela Superior de Guerra. Consultor del Centro Regional de las Naciones Unidad contra Tráfico de Armas, Municiones y Explosivos, el Desarrollo y la Paz para Latinoamérica y el Caribe. Correo electrónico: ardilac@esdegue.mil.co

Master in Relations and International Business of Nueva Granado Military University, Specialist in Political Studies of EAFIT University, Specialist in Security and Defense of the War College and Professional in Security Management and Socio-Political Analysis of the Intelligence School of the Army. Professor in the Master in National Security and Defense of the War College. Consultant for the Regional Center of the United Nations against Traffic of Armament, Ammunition, and Explosives, Development and Peace for Latin America and the Caribbean.E-mail:ardilac@esdegue.mil.co

\author{
Argentina's Strategic interests in \\ The Institutional Framework of Mercosur ${ }^{2}$
}

Carlos Alberto Ardila Castro ${ }^{3}$
Resumen: En América Latina la formulación de políticas de seguridad y defensa diseñadas por los Estados, tienen en cuenta diversos factores dentro de los que se incluyen elementos relacionados con la geopolítica, la política interna, las amenazas transnacionales, la transformación estratégica de la región y la política de seguridad hemisférica de los Estados Unidos. De acuerdo a esto, la evolución en términos de disposición de fuerzas militares, la compra y venta de armamento y la búsqueda de mecanismos de cooperación, obedecen a la identificación de prioridades e intereses estratégicos derivados de contextos que los Estados perciben como fuentes de vulnerabilidad, a partir de los cuales implementan estrategias de mitigación que les permitan la obtención de su interés nacional. Este artículo tiene como objetivo analizar la importancia de Mercosur para Argentina como materialización de una política de seguridad, teniendo en cuenta sus intereses estratégicos y las amenazas que el Estado identifica como focos de vulnerabilidad.

Palabras clave: Interés estratégico, Mercosur, política exterior Argentina, seguridad regional.

Abstract: The formulation of security and defense policies designed by the states, have into account different factors among which include elements related with geopolitics, domestic politics, transnational threats, strategic transformation of the region and the hemispheric security policy of the United States. According to this, the evolution in terms of provision of military forces, the purchase and sale of armament and the search for mechanisms of cooperation, are based on the identification of priorities and strategic interests form contexts perceived by the states as sources of vulnerability, from which the States implement mitigation strategies in order to obtain their national interest. This article aims to analyze the importance of Mercosur for Argentina as a security policy, taking into account its strategic interests and threats identified by the State as vulnerabilities.

Key Words: Foreign policy of Argentina, Mercosur, Strategic interest, Regional Security. 


\section{Introducción}

En América Latina la formulación de políticas de seguridad y defensa ${ }^{4}$ diseñadas por los Estados, tienen en cuenta diversos factores dentro de los que se incluyen elementos relacionados con la geopolítica, la política interna, las amenazas transnacionales, la transformación estratégica de la región y la política de seguridad hemisférica de los Estados Unidos. De acuerdo a esto, la evolución en términos de disposición de fuerzas militares, la compra y venta de armamento y la búsqueda de mecanismos de cooperación, obedecen a la identificación de prioridades derivadas de contextos que los Estados perciben como fuentes de vulnerabilidad, a partir de los cuales implementan estrategias de mitigación que les permitan la obtención de su interés nacional.

Este artículo tiene como objetivo analizar la importancia de Mercosur para Argentina como materialización de una política de seguridad, teniendo en cuenta sus intereses estratégicos y las amenazas que el Estado identifica como focos de vulnerabilidad. Para llevar a cabo este propósito, inicialmente se abordan los intereses estratégicos de Argentina en términos de seguridad y defensa, para después hacer referencia a Mercosur como un mecanismo a través del cual se aporta a la satisfacción de dichos intereses. Finalmente, el artículo plantea una reflexión acerca de las lecciones que pueden ser aprendidas por Colombia y los beneficios que generaría para su política exterior.

Es importante destacar que la temática propuesta es considerada desde una perspectiva teórica constructivista, dado que ésta permite abordar la cooperación a partir de una concepción integral, en dónde se involucran las identidades e intereses de los Estados como variables explicativas de la acción colectiva (Schünemann, 2006).

\section{Método}

La investigación se desarrolla dentro de la línea Modelos en Seguridad y Defensa, el cual realizó un estudio de caso de los diferentes modelos que los Estados de Suramérica aplican para desarrollar su política de Seguridad y Defensa. Se buscó realizar un análisis cualitativo teniendo como referencia los marcos normativos de cada uno

${ }_{4}^{4}$ "Política de defensa" se define como "el conjunto de principios y criterios con que el Estado orienta su función de defensa con vistas a preservar la soberanía e integridad del territorio y contribuir al logro de los demás objetivos nacionales" (Derghougassian, 2012, p. 13). de los países y los diferentes conceptos que cada uno tiene de la seguridad y defensa, comparando los resultados con los utilizados por el Estado Colombiano.

\section{Intereses estratégicos de Argentina: un aná- lisis desde el ámbito de la seguridad}

Los intereses, entendidos bajo la perspectiva teórica constructivista como schemas "or scripts, frames, or representations, which are knowledge structures that "make possible the identification of objects and events" (Wendt, 1999, p.122) hacen referencia a objetivos deseables que motivan determinada acción y que se organizan jerárquicamente en relación con la identidad de un Estado. Así pues, los intereses se caracterizan por una condición de evolución, determinada por una multiplicidad de factores, dentro de los cuales se incluyen los procesos de aprendizaje derivados de la historia de cada sociedad.

Este apartado tiene como propósito señalar los intereses estratégicos de Argentina, teniendo en cuenta sus características geopolíticas y la evolución de las políticas de seguridad de acuerdo a los acontecimientos más importantes durante y después de la dictadura militar, uno de los cuales fue el uso de la fuerza como medio para la reclamación de las Islas Malvinas. En este sentido, inicialmente se hará referencia a la Guerra de las Malvinas, para después analizar los intereses actuales y la importancia de los mecanismos de cooperación para el cumplimiento de los mismos.

En el caso de Argentina, los intereses y las políticas de defensa y seguridad han estado enmarcados en la garantía de un sistema democrático, a partir del cual se busca prevenir el resurgimiento de un régimen autoritario (Diamint, 1992, p. 2). Teniendo en cuenta las consecuencias derivadas del gobierno militar y los efectos generados en términos de la fragmentación social. Así pues, desde la presidencia de Carlos Menem se estableció como interés fundamental la implementación de políticas dirigidas a la disminución de la posibilidad de nuevos levantamientos y la coordinación de acuerdos con las fuerzas militares implicadas en el proceso de reorganización nacional ${ }^{5}$.

${ }^{5}$ El proceso de reorganización nacional hace referencia al periodo de la dictadura militar que se desarrolló en Argentina durante 1976 y 1983, a partir del cual se establece una junta militar cuyo principal propósito era la instauración de una nación católica (Saborido, 2005, p. 249). 
Bajo este contexto de dictadura militar, otro acontecimiento que fue determinante en la historia Argentina y que marcó un precedente en cuanto a los patrones de comportamiento y la concepción identitaria del Estado, fue la Guerra de las Malvinas. El interés estratégico en dichas islas, radica en que en tiempos de guerra, el dominio de éstas representa el control de los océanos, dado que se encuentra ubicada en el cruce de las vías marítimas que unen al Atlántico con el Pacífico en el hemisferio austral. Asimismo, es importante señalar que las islas tiene una importante capacidad como plataforma logística, y son idóneas para interceptar las ondas hertzianas y electromagnéticas sobre América del Sur. Además de la posibilidad de explotación de hidrocarburos y establecimiento de una base para un posible reclamo sobre la Antártida (Razoux, 2002, p.10).

El impacto identitario de la Guerra de las Malvinas, surge a partir del hipernacionalismo generado desde 1976, época en la cual la junta militar tomó el poder en Buenos Aires y más específicamente en 1981, cuando en cabeza del General Leopoldo Galtieri ${ }^{6}$, el gobierno militar se propone la meta simbólica de la reconquista de las islas, las cuales habían sido controladas por más de 150 años por el Reino Unido (Razoux, 2002, p.11).

Es así como, en abril de 1982, después de 17 años de conversaciones infructuosas, Argentina tomó la decisión de recuperar las Islas Malvinas por la fuerza, territorio que en ese momento era considerado como un área en disputa por parte de las Naciones Unidas (Camogli, 2007). La Guerra termina a causa de la victoria militar inglesa y la rendición argentina, situación que no representó la solución de la controversia territorial, dado que de acuerdo con el Derecho Internacional, la victoria militar no constituye un medio legítimo para la adquisición de territorio. En este sentido, "la fuerza solamente determinó la posesión de las islas: el reclamo subyacente sobre la propiedad persiste" (Aizenstatd, 2012, p.99).

De acuerdo con lo anterior, la derrota en la Guerra de las Malvinas representó un punto de inflexión en la política de defensa argentina, demostrando "la incompetencia de la dictadura en la lectura que había hecho de la realidad internacional, sus supuestos erróneos a la hora de decidir lanzar una operación que buscaba golpear para negociar, y sobre todo, la falta de preparación de la Aviación, la Marina y el Ejército para en- frentar una potencia mundial de segundo rango como era Gran Bretaña" [curviva incluida] (Derghougassian, 2012, p. 13).

Bajo este contexto, se generó un debate acerca de la prohibición de la amenaza o el uso de la fuerza como un principio inviolable del derecho internacional, el cual fue reafirmado por diferentes países y Organizaciones, sin que éstos se pronunciaran sobre la legitimidad de la reclamación territorial.

Así pues, dada la percepción negativa de Argentina en el escenario internacional, después del proceso de Reorganización Nacional, el país estableció como propósito fundamental la subordinación de las fuerzas militares al poder civil, así como la alineación con los asuntos estratégicos globales y el compromiso con la estabilidad regional en términos de la seguridad militar y la adecuada respuesta a los desafíos transnacionales.

Actualmente, los intereses estratégicos de Argentina se consolidan a partir no sólo de los factores vigentes que caracterizan el contexto de seguridad, también de los procesos de aprendizaje y la evolución que ha tenido la forma de percepción subjetiva del país en la región, y la identificación de las dinámicas y actores que afectan el escenario internacional.

Es así como en el Libro Blanco de la Defensa, publicado en el año 2010, se señalan las características y apreciación del Sistema Internacional y se destacan los siguientes fenómenos: "el protagonismo ascendente de actores de naturaleza no estatal o transnacional; la comprensión multidimensional de la problemática de la seguridad y la dificultad en la construcción de conceso global para la respuesta a novedosos desafíos colectivos" (Ministerio de Defensa, 2010, p. 21). A partir de esto, se evidencia cómo la cooperación es empleada como un concepto recurrente e identificada como un elemento estratégico para la mitigación de amenazas y el aprovechamiento de oportunidades, en un escenario en dónde la impredecibilidad y los desafíos transnacionales se establecen como factores característicos.

Así las cosas, los intereses van a hacer referencia a diferentes ámbitos que involucran la esfera geopolítica, cultural, económica y social. Sin embargo, para efectos del desarrollo de este documento se van a mencionar por un lado los intereses vitales, y por otro lado, los intereses estratégicos.

${ }^{6}$ Comandante de la época del Ejército argentino. 
CIENCIA Y PODER AÉREO | Revista Científica de la Escuela de Postgrados de la Fuerza Aérea Colombiana | Vol. 9 | Enero - Diciembre de 2014

Con respecto a los primeros, éstos aluden a aquellos intereses que afectan la supervivencia y estabilidad de la nación, y que por su alto grado de importancia tienen un carácter de inmutabilidad a lo largo del tiempo. De acuerdo a esto, los intereses vitales son:

- La soberanía e independencia de la Nación Argentina.

- Su integridad territorial.

- Su capacidad de autodeterminación.

- La protección de la vida y la libertad de sus habitantes (RESDAL, s.f., p. 7).

En lo que se refiere a los intereses estratégicos, es importante destacar la preservación de la paz y la seguridad internacional; la integración y seguridad regional; la preservación de vacíos geopolíticos; el espacio aéreo argentino; los espacios interoceánicos y la preservación del posicionamiento argentino en el Sistema Antártico (RESDAL, s.f., p. 8).

De acuerdo a esto, aunque Argentina se establece como un país con unas características geopolíticas favorables, teniendo en cuenta que es uno de los Estados con mayor superficie del mundo, cuyos extremos tocan 3 océanos, y con una destacada importancia continental derivada de su identificación como un espacio de convergencia entre los países que integran el Cono Sur Americano, existen vulnerabilidades de diferente índole que afectan el cumplimiento de los intereses estratégicos (Koutoudijan, 2004, p. 510) (Ver Tabla 1).

\section{Tabla 1. Características geopolíticas argentinas}

1. Península templada en el Hemisferio sur

2. Octavo país del mundo en extensión

3. Población étnicamente homogénea

4. Posición excéntrica al grueso del tráfico mundial

5. Oposición de estaciones climáticas con el Hemisferio norte

6. País Atlántico por su geografía y su historia

7. País Antártico por su geografía

8. País de convergencias de tráficos y culturas

9. País-síntesis en lo histórico-cultural con tendencia a ser un mosaico cultural
10. Climas templados en todas sus variaciones con gran potencialidad productiva

11. $700.000 \mathrm{~km} 2$ de pampa húmeda y 1.2 millones de $\mathrm{km} 2$

12. Superficie agropecuaria de 279 millas. Has.

13. Estructura económica regional fuertemente desequilibrada

14. País de mediano desarrollo con estructura social de gran clase media, aunque con marcada regresión los últimos 25 años

15. País de mediano desarrollo por su PBN, su patrimonio geográfico y el IDH

Fuente: Koutoudijan (2004b). Prospectiva Geopolítica Argentina. En H. Gastón, Lecciones de geopolítica, pp. 491-553. Buenos Aires: Centro Ficch.

Dentro de dichas vulnerabilidades se incluyen condiciones geográficas tales como la amplia extensión terrestre, marítima y aérea; la posibilidad de aislamiento de los extremos del territorio nacional; la ausencia de un control efectivo del Atlántico Sud-occidental y la Antártida, y la falta de conectividad interregional (Koutoudijan, 2004).

Así pues, los principales problemas relacionados con la seguridad nacional, siguen estando focalizados en disputas internacionales, específicamente en las pretensiones sobre las Islas Malvinas; la reclamación territorial en la Antártida; la delimitación de la frontera en el Campo de Hielo Sur y las dificultades derivadas del contrabando y el tráfico ilícito de estupefacientes en las zonas porosas de la frontera con Bolivia (Central Intelligence Agency, 2014). En términos de drogas ilícitas, Argentina se identifica por ser un país importante en el tránsito de la cocaína que va rumbo a Europa y por tener actividades de lavado de dinero en la Triple Frontera (Central Intelligence Agency, 2014).

Teniendo en cuenta el panorama expuesto anteriormente, Argentina debe establecer estrategias que consideren la condición transnacional de las amenazas que caracterizan al Sistema Internacional, y las vulnerabilidades que afectan su interés estratégico en el ámbito regional, con el fin de consolidar mecanismos a través de los cuales se genere una mitigación efectiva de las mismas. En el próximo apartado se hará alusión al Mercosur como materialización de una política de seguridad Argentina. 


\section{Los intereses estratégicos de Argentina en el marco institucional de MERCOSUR}

El Mercosur es una iniciativa de cooperación integrada por Argentina, Brasil, Paraguay, Uruguay, Venezuela y Bolivia, y se crea a partir de la identificación de valores compartidos expresados en "sus sociedades democráticas pluralistas, defensoras de las libertades fundamentales, de los derechos humanos, de la protección del medio ambiente y del desarrollo sustentable, así como su compromiso con la consolidación de la democracia, la seguridad jurídica, el combate a la pobreza y el desarrollo económico y social con equidad" (Mercosur, 2014, p 3).

La identificación de estos valores como pilares de construcción del Mercosur, evidencia la importancia de las identidades e intereses como condiciones determinantes para la consolidación de escenarios de cooperación, identificada como una acción colectiva, en dónde se da un proceso de creación de nuevas definiciones y se establece un marco de interpretación común de la realidad (Schünemann, 2006).

En este sentido, además del reconocimiento de valores compartidos, el Tratado de Asunción señala como intereses u objetivos fundamentales del Mercosur:

1. La libre circulación de bienes, servicios y factores productivos entre los países, a través, entre otros, de la eliminación de los derechos aduaneros y restricciones no arancelarias a la circulación de mercaderías y de cualquier otra medida equivalente;

2. el establecimiento de un arancel externo común y la adopción de una política comercial común con relación a terceros Estados o agrupaciones de Estados y la coordinación de posiciones en foros económicos comercial regionales e internacionales;

3. la coordinación de políticas macroeconómicas y sectoriales entre los Estados Partes: de comercio exterior, agrícola, industrial, fiscal, monetario, cambiario y de capitales, de servicios, aduanera, de transportes y comunicaciones y otras que se acuerden, a fin de asegurar condiciones adecuadas de competencia entre los Estados Partes;

4. el compromiso de los Estados Partes de armonizar sus legislaciones en las áreas pertinentes, para lograr el fortalecimiento del proceso de integración (Mercosur, 2014, p.).

En este orden de ideas, el Mercosur se crea con un propósito dirigido fundamentalmente al ámbito económico. Sin embargo, dados los desafíos transnacionales que afectan la región Sudamericana, surge la necesidad de implementar marcos de seguridad que tengan en cuenta las especificidades de la región, y que aporten a un desarrollo de la misma con base en una interpretación común del contexto que la afecta.

Es así como el establecimiento de un pacto de defensa y seguridad en el marco de la estructura institucional del Mercosur, debe estar dirigida a la "conservación de la soberanía e integridad territorial de los Estados de la región, al cuidado de sus recursos naturales para permitir un mayor bienestar del pueblo y a un grado suficiente de decisión en el concierto de competencias interbloque que se avizoran en el Siglo XXI" (Koutoudijan, 2004a, p. 5).

En la medida en que se logre articular un acuerdo de seguridad que plantee soluciones construidas sobre la identificación de problemas compartidos, y se avance hacia la institucionalización de medidas concertadas, va a ser posible que los países partícipes del acuerdo, dentro de los cuales está Argentina, logren diseñar estrategias integrales que permitan controlar los problemas asociados al tráfico de drogas y el terrorismo, y a su vez aporten al fortalecimiento de una visión de región que asegure la protección de sus recursos naturales y reduzca la vulnerabilidad en relación con factores externos.

En este sentido, es pertinente destacar cuatro esferas de acción en las que aportaría un pacto de defensa y seguridad en el contexto de Mercosur:

1. Disminución de la probabilidad de desintegración regional por acción de poderes exógenos, situación que se podría presentar a causa de un cambio en la estructura política internacional o de la multiplicación de conflictos por recursos naturales.

2. Disminución de las vulnerabilidades del espacio Mercosur, algunas de las cuales son: la desprotección de la plataforma submarina Sudamericana; el espacio aéreo y exterior de la región; los espacios semivacíos terrestres 
CIENCIA Y PODER AÉREO | Revista Científica de la Escuela de Postgrados de la Fuerza Aérea Colombiana |Vol. 9 | Enero - Diciembre de 2014

como Amazonía, Chaco Boreal y Austral, Patagonia y Antártida Sudamericana; los grandes ecosistemas regionales, entre otros.

3. Proyección política del peso específico de la región

4. Mayor participación en calidad de bloque regional en escenarios internacionales como la ONU o la OEA (Koutoudijan, 2004a, p. 4).

Así pues, considerando las esferas que se ven beneficiadas a partir de un acuerdo de cooperación en materia de seguridad, es evidente que dicho acuerdo aporta a la consecución de los intereses estratégicos de Argentina, teniendo en cuenta que en la actualidad la mayoría de problemas para el país están relacionados con disputas territoriales internacionales y con la ausencia de un control efectivo de vacíos geopolíticos.

En relación con lo anterior, es importante destacar el "Acuerdo Marco sobre Cooperación en materia de Seguridad Regional entre los Estados partes del Mercosur, la República de Bolivia, la República de Colombia, la República de Chile, la República del Ecuador, la República del Perú y la República Bolivariana de Venezuela" (Mercosur, 2006, p. 7). Este acuerdo tiene como objetivo:

optimizar los niveles de seguridad de la región, promoviendo la más amplia cooperación y asistencia recíproca en la prevención y represión de las actividades ilícitas, especialmente las transnacionales, tales como: el tráfico ilícito de estupefacientes y sustancias psicotrópicas, el terrorismo internacional, el lavado de activos, el tráfico ilícito de armas de fuego, municiones y explosivos, el tráfico ilícito de personas, el contrabando de vehículos y los daños ambientales, entre otras (Mercosur, 2006, p. 8).

Para dar cumplimiento a este objetivo, se toma a la cooperación como principal instrumento, a través del intercambio de información, la realización de actividades operativas coordinadas y el establecimiento de mecanismos que materialicen los esfuerzos comunes en el ámbito de la seguridad pública y la seguridad de las personas y sus bienes.

Es importante destacar que de la nómina de las Fuerzas de seguridad y/o policiales comprometidas en el acuerdo, Argentina es el país con mayor cantidad de sectores involucrados, a saber: Gendarmería Nacional Argentina; Prefectura Naval Argentina; Policía Federal Argentina y Policía de seguridad Aeroportuaria. Dicha condición, evidencia la importancia que constituye el Mercosur como un marco para la implementación de políticas de seguridad, de acuerdo a sus intereses y a la percepción de amenaza frente a los mismos.

Sin embargo, a pesar del mencionado acuerdo, el avance a la consolidación de un pacto de defensa y seguridad, se ha visto subordinado frente al establecimiento de medidas de confianza mutua, que no se ven traducidas en una armonización de políticas de defensa (Riquelme, 2011, p. 29). Esta situación afecta la capacidad competitiva de Mercosur en términos de las propuestas y la influencia que puede generar como bloque, para la búsqueda de soluciones efectivas a problemas transnacionales no sólo en el ámbito regional, también en el escenario internacional.

\section{Colombia y Mercosur}

Dentro del marco institucional de Mercosur, Colombia se identifica como un país en calidad de asociado y se ha destacado por su papel dirigido al fomento de la integración regional con otros mecanismos tales como la Comunidad Andina (CAN) (Ministerio de Relaciones Exteriores-Colombia, 2014).

Bajo esta perspectiva, es pertinente mencionar que Colombia se destaca por tener una posición geográfica favorable, teniendo en cuenta que participa de la cuenca del Gran Caribe, la cuenca del Pacífico y el espacio Sudamericano y amazónico, lo cual lo sitúa en el corazón del continente y lo proyecta como un país con la capacidad de generar puentes entre diferentes zonas geográficas.

Así pues, el involucramiento en diversos mecanismos de cooperación, permite que Colombia siga promoviendo una visión de política exterior basado en una condición de Respice Omnia, referido al principio de mirar al universo y al conjunto (Fernández, 2012, p. 71), considerando el ambiente de interdependencia que caracteriza el Sistema Internacional y la posibilidad de inserción que ofrecen estos espacios de concertación.

Sin embargo, la ausencia de la identificación de unos intereses estratégicos puntuales, basados en análisis geopolíticos y de vulnerabilidades y posibilidades de aprovechamiento de oportunidades, de acuerdo a 
las condiciones internas y externas, hacen que Colombia no emplee los mecanismos de cooperación como herramientas para la proyección de su interés nacional, priorizando así comportamientos pragmáticos. A pesar de que dichos comportamientos se establecen como canales efectivos para el cumplimiento de determinados objetivos, no aportan a una visión de mediano y largo plazo que permita la consolidación de políticas de Estado, a partir de las cuales se favorezca la posición de Colombia en el escenario internacional.

En términos de la cooperación con Mercosur, Colombia podría profundizar su participación en este mecanismo, a través de su experiencia en aspectos de seguridad, tales como el control del tráfico de armas, municiones y explosivos, así como los problemas asociados con la producción y distribución de droga. En este sentido, se proyectaría a Colombia no sólo como un país receptor de ayuda internacional, también como un actor propositivo frente a los temas de interés mundial.

\section{Conclusiones}

Las políticas de seguridad de Argentina y sus intereses estratégicos, obedecen a un análisis de sus prioridades en relación con las condiciones del sistema internacional. Así pues, en el proceso de construcción de estos elementos, intervinieron tanto los recursos cognitivos aprendidos a partir de los acontecimientos que han marcado la historia de Argentina como un país con alta visibilidad en el espacio latinoamericano, como las aspiraciones y las amenazas que existen frente a las mismas en un contexto de desafíos transnacionales.

Actualmente, algunos de los principales intereses estratégicos de Argentina son: la integración y seguridad regional; la preservación de vacíos geopolíticos; el espacio aéreo argentino; los espacios interoceánicos y la preservación del posicionamiento argentino en el Sistema Antártico. Es así, como Mercosur se establece como un marco institucional pertinente para promover un pacto de defensa y seguridad, por medio del cual se busque la conservación de la soberanía e integridad territorial de los Estados de la región, así como el cuidado de sus recursos naturales, condición que representa una prioridad para Argentina, cuyos principales problemas relacionados con la seguridad nacional, siguen estando focalizados en disputas internacionales.

Sin embargo, aún está por verse que tanto avanza Mercosur en términos de la consolidación de un pacto de defensa y seguridad, teniendo en cuenta lo mencionado en apartados anteriores, hasta el momento se han privilegiado el establecimiento de medidas de confianza mutua, lo que no ha permitido una armonización efectiva de políticas de defensa.

\section{Referencias}

Aizenstatd, A. (2012). A treinta años de la guerra: las islas Malvinas y los principios de Derecho Internacional. Estudios Internacionales 173, pp. 91-116.

Camogli, P. (2007). Batallas de Malvinas. Buenos Aires: Aguilar.

CIA. (2014). The World Factbook Argentina. [en línea], disponible en: https://www.cia.gov/library/publications/the-worldfactbook/geos/ar.html, recuperado el 27 de Febrero de 2014.

Derghougassian, K. (2012). La Defensa en el Siglo XXI. Argentina y la seguridad regional. Buenos Aires: Capital Intelectual.

Diamint, C. (1992). Cambios en la política de seguridad. Argentina en busca de un perfil no conflictivo. FASOC Vol. VII, (1), pp. 1-16.

Fernández, G. (2010). El universo es el límite: el futuro de la política exterior Colombiana. Bogotá: Universidad del Rosario.

Koutoudijan, A. (2004a). Pacto de defensa y Sseguridad del Cono Sur Sudamericano. En Stanganelli, I. (2005). Seguridad y Defensa en el Cono Sur, pp. 191-221. Mendoza: Inca.

Koutoudjian, A. (2004b). Prospectiva Geopolítica Argentina. En Gastón, H. (2004). Lecciones de geopolítica, pp. 491-553. Buenos Aires: Centro Ficch.

Ministerio de Defensa. (2010). Libro Blanco de la Defensa, pp. 1-404. [En línea] disponible en: HYPERLINK"http://www.mindef.gov. ar/libro_blanco/Libro_Blanco_de_la_Defensa_2010-FeDeErratas.pdf" http://www.mindef.gov.ar/libro_blanco/Libro_ Blanco_de_la_Defensa_2010-FeDeErratas.pdf Recuperado: 27 de febrero de 2014

Ministerio de Relaciones Exteriores. (2014). Mercado Común del Sur-Mercosur. [En línea] disponible en: http://www.cancilleria.gov.co/international/consensus/mercosur, recuperado el 27 de Febrero de 2014.

Mercosur. (2006). Acuerdo Marco sobre Cooperación en Materia de Seguridad Regional entre los Estados partes del Mercosur, la República de Bolivia, la República de Colombia, la República de Chile, la República del Ecuador, la República del Perú y la República Bolivariana de Venezuela. [En línea] disponible en: http://www.sice.oas.org/trade/mrcsrs/decisions/dec1606s.pdf, recuperado: 27 de febrero de 2014.

Mercosur. (2014). Tratado de Asunción. [En línea] disponible en: $\quad$ http://www.mercosur.int/innovaportal/file/719/1/ CMC_1991_TRATADO_ES_Asuncion.pdf, recuperado el 27 de Febrero de 2014

Razoux, P. (2002). La Guerra de las Malvinas. Istor, pp. 9-28.

RESDAL. (s.f.). Argentina. Libro Blanco de la Defensa Nacional - Parte III: las políticas de defensa. [En línea] disponible en: http://www.resdal.org/Archivo/defa-plll.htm http://www. resdal.org/Archivo/defa-plll.htm, recuperado el 27 de Junio de 2013. 
CIENCIA Y PODER AÉREO | Revista Científica de la Escuela de Postgrados de la Fuerza Aérea Colombiana | Vol. 9 | Enero - Diciembre de 2014

Saborido, J. (2005). El nacionalismo argentino en los años de plomo: la revista cabildo y el proceso de reoganización nacional (1976-183). Anuario de estudios americanos, pp. 235-270.

Schünemann, J. (2008). Algo más que racional: la dimensión social de la cooperación interregional y el interregionalismo en las relaciones internacionales. Documentos de Política Pública: las memorias de los foros 2008, pp. 165-219.

Wendt, A. (1999). Social Theory of International Politics. NY: Cambridge University Press.

Bibliografía consultada / No citada

Acuña, C. (1993). Argentina. Hacia un nuevo modelo. Nueva Sociedad No 126, pp.11-24.

Crisóstomo, C. (2009). UNASUR y la proyección del Consejo de Seguridad Suramericano. UNISCI, pp. 62-78. [En línea] disponible en: http://merln.ndu.edu/whitepapers/Argentina2001 print.pdf, recuperado el 04 de Julio de 2013.

Rivas, S., y Cicalesi, J. (2009). Panorama de la Defensa en Argentina. Tecnología Militar, pp. 8-10.

Scheetz, T. (2005). Evolución de los presupuestos de defensa de los países sudamericanos. Tecnologia Militar, pp. 12-13.

Tapia, G. (2005). La Seguridad Sudamericana y el Rol del Sector de la Defensa. Tecnología Militar, pp. 6-7. 